\title{
Practical Algebraic Side-Channel Attacks against ACORN
}

\author{
Alexandre Adomnicai $^{1,2}$ Laurent Masson ${ }^{1}$ Jacques J.A. Fournier ${ }^{3}$ \\ ${ }^{1}$ Trusted Objects \\ ${ }^{2}$ Mines Saint-Étienne \\ ${ }^{3}$ Univ. Grenoble Alpes, CEA-Leti
}

$21^{\text {th }}$ International Conference on Information Security \& Cryptology

Seoul, November $30^{\text {th }} 2018$

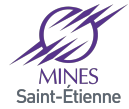




\section{Competition for Authenticated Encryption: Security, Applicability, and Robustness (CAESAR)}

$\triangle$ New authenticated encryption schemes that offer advantages over AES-GCM

$\triangleright$ At the end, a multiple-algorithm portfolio

$\triangleright 57$ candidates for the first round (March 2014)

$\triangleright 7$ finalists (March 2018)

Lightweight applications

ACORN [Wu14] Ascon
High-performance applications
AEGIS MORUS
OCB

Nonce misuse resistance

COLM

Deoxys-II

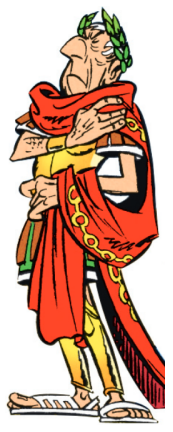




\section{Lightweight Applications Criteria}

$\triangleright$ Fits into small hardware area and/or small code for 8-bit CPUs

$\triangleright$ Natural ability to protect against side-channel attacks (SCA)

$\triangleright$ Good performance in both software and hardware (especially energy/bit)

$\triangleright$ Low overhead for short messages (e.g. 16 bytes) 


\section{Side-Channel Attacks (SCA)}

$\triangleright$ Cryptographic primitives are designed to be finally executed on a physical system

$\triangleright$ The physical characteristics of the computing platform produce side effects depending on the processed data

- Power consumption $\circ$ Electromagnetic emanations (EM) ○ Time execution ...

$\triangleright$ One can measure these side effects to get information on the processed values during sensitive operations

$\triangleright$ Using an appropriate leakage model, one can recover the secrets involved in calculations

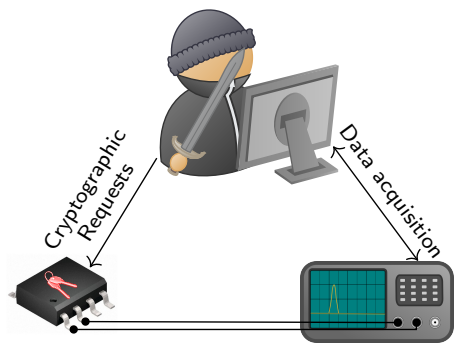




\section{Susceptibility of ACORN Towards SCA}

$\triangleright$ No conditional branch or use of look-up table $\Rightarrow$ intrinsic constant-time implementation

$\triangleright 2$ previous works regarding power/EM side-channels

1 - Leakage detection on hardware implementation using t-test $\left[\mathrm{DAF}^{+} 18\right]$

2 - Theoretical attack using differential power analysis (DPA) [AFM18]

$\triangleright$ Assessment leakage methodologies can be insufficient to guarantee the security level of an implementation $\Rightarrow$ need to run additional tests

$\triangleright$ No practical DPA against ACORN published to date $\Rightarrow$ how hard is it to put into practice? 


\section{Previous Work (Leakage Detection)}
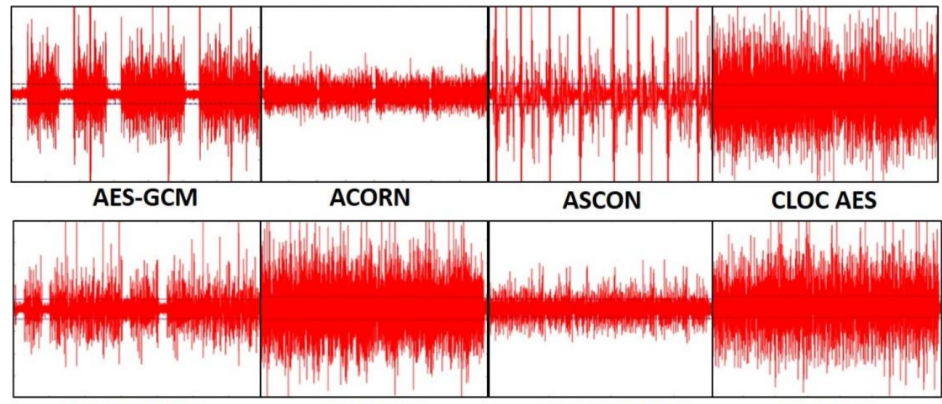

CLOC TWINE

SILC AES

SILC PRESENT

SILC LED

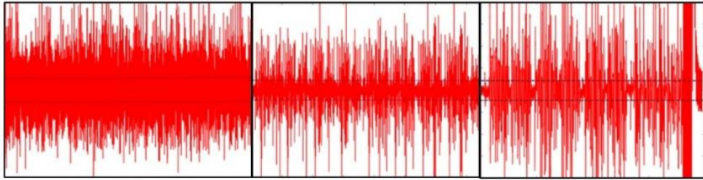

JAMBU AES

JAMBU SIMON

KETJE JR 


\section{Our Contribution}

$\triangleright$ Put into practice the theoretical attack described in [AFM18]

$\triangleright$ Explicit definition of all selection functions required to mount the attack

$\triangleright$ Proposition of 2 different optimizations of the reference attack

1 - Maximize the practical efficiency

2 - Minimize the number of IV bits to consider 


\section{ACORN in a nutshell}

$\triangleright$ Authenticated stream cipher introduced by Hongjun

Wu in 2014

$\triangleright$ 128-bit key, 128-bit IV, 128-bit tag

\begin{tabular}{l}
\hline StateUpdate $(S, m)$ \\
\hline$S_{289} \leftarrow S_{289} \oplus S_{235} \oplus S_{230}$ \\
$S_{230} \leftarrow S_{230} \oplus S_{196} \oplus S_{193}$ \\
$S_{193} \leftarrow S_{193} \oplus S_{160} \oplus S_{154}$ \\
$S_{154} \leftarrow S_{154} \oplus S_{111} \oplus S_{107}$ \\
$S_{107} \leftarrow S_{107} \oplus S_{66} \oplus S_{61}$ \\
$S_{61} \leftarrow S_{16} \oplus S_{23} \oplus S_{0}$ \\
$k s \leftarrow \kappa(S)$ \\
$c \leftarrow k s \oplus m$ \\
$f \leftarrow(S, k s)$ \\
for $i$ from 0 to 291 do \\
$\quad S_{i} \leftarrow S_{i+1}$ \\
$S_{292} \leftarrow f \oplus m$ \\
\hline
\end{tabular}

$\triangleright$ Feedback bit $f$ and keystream $k s$ are computed in

$\triangleright$ Based on the concatenation of 6 LFSRs (+ a 4-bit register)

$\triangleright$ Encryption and authentication share the same state (293-bit long) a nonlinear manner

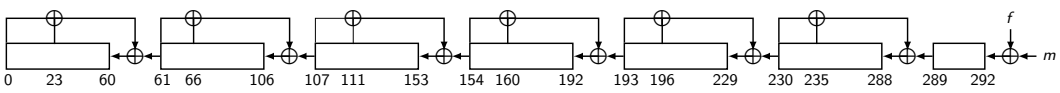




\section{Differential Power Analysis (DPA)}

$\triangleright$ Power consumption of an operation varies with the operand values $\Rightarrow$ intermediate values leak

$\triangleright$ Variations are relatively small, not directly observable $\Rightarrow$ use of statistical tools over several measurements

$\triangleright$ Targeted intermediate values are defined by selection functions $y=\varphi(m, k)$

$\triangleright$ It is assumed that $m$ varies over measurements and is known by the attacker

Built from key bits Built from input bits Built from key and input bits 


\section{Theoretical DPA against ACORN}

$\triangleright$ DPAs against stream ciphers usually focus on the initialization phase

IV bits do not directly interact with key bits but with feedback bits

$\triangleright$ The theoretical attack targets intermediate bits $y_{i}=f_{128+i} \oplus / V_{i}$

$$
\begin{aligned}
& \text { AcornInit }(S, K, I V) \\
& \hline\left(S_{0}, \ldots, S_{292}\right) \leftarrow(0, \ldots, 0) \\
& \text { for } i \text { from } 0 \text { to } 127 \text { do } \\
& \quad S \leftarrow \text { StateUpdate }\left(S, K_{i}\right) \\
& \text { for } i \text { from } 0 \text { to } 127 \text { do } \\
& \quad S \leftarrow \text { StateUpdate }\left(S, / V_{i}\right) \\
& S \leftarrow \text { StateUpdate }\left(S, K_{0} \oplus 1\right) \\
& \text { for } i \text { from } 1 \text { to } 1535 \text { do } \\
& \quad S \leftarrow \text { StateUpdate }\left(S, K_{i \bmod 128)}\right.
\end{aligned}
$$
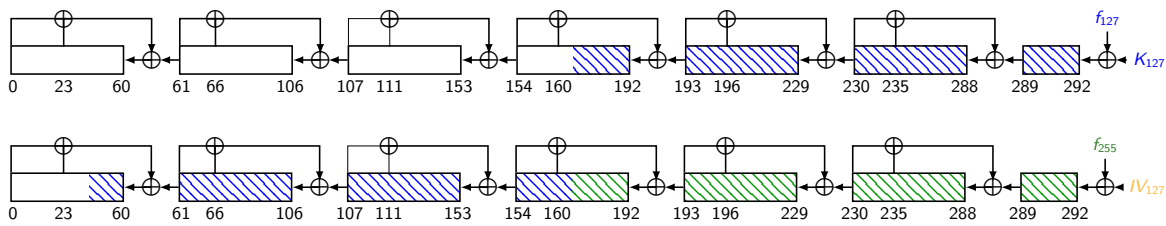


\section{Theoretical DPA against ACORN}

$\triangleright f_{128+i}$ are defined by key bit combinations: $f_{128}=\left(\neg K_{70} \oplus K_{11} \oplus K_{16}\right) \wedge \neg K_{28} \oplus K_{31}$

$\triangleright$ Returns a system of equations $\mathscr{F}$ to solve $\Rightarrow$ algebraic side-channel attack!

$\triangleright$ Bad news: from $i=177, f_{i}$ are not only key-dependent anymore

$\triangleright \mathscr{F}_{[0,48]}$ has 49 equations for 128 unknowns $\Rightarrow$ too many solutions...

$$
\begin{aligned}
f_{177} & =\neg\left(\left(\neg\left(\left(\neg K_{70} \oplus K_{11} \oplus K_{16}\right) \wedge \neg K_{28}\right) \oplus \neg K_{31} \oplus / V_{0} \oplus K_{69} \oplus K_{10} \oplus K_{15} \oplus K_{74}\right.\right. \\
& \left.\left.\oplus K_{15} \oplus K_{20}\right) \wedge\left(\neg K_{44} \oplus K_{7} \oplus K_{10} \oplus K_{5} \oplus K_{11}\right)\right) \oplus\left(\neg \left(\left(\neg K_{61} \oplus K_{2} \oplus K_{7}\right)\right.\right. \\
& \left.\left.\wedge \neg K_{19}\right) \oplus \neg K_{22} \oplus K_{119} \oplus K_{60} \oplus K_{1} \oplus K_{65} \oplus K_{11}\right) \wedge\left(K_{76} \oplus K_{17} \oplus K_{22} \oplus K_{40}\right. \\
& \left.\oplus K_{3} \oplus K_{43} \oplus K_{9} \oplus K_{38} \oplus K_{1} \oplus K_{4} \oplus K_{5} \oplus K_{44} \oplus K_{7} \oplus K_{8} \oplus K_{5} \oplus K_{11}\right) \\
& \oplus \neg K_{38} \oplus K_{1} \oplus K_{4} \oplus K_{5} \oplus K_{80} \oplus K_{21} \oplus K_{26} \oplus K_{43} \oplus K_{6} \oplus K_{46} \oplus K_{12}
\end{aligned}
$$




\section{Distributive property of AND over XOR to the rescue!}

$$
\begin{aligned}
f_{177} & =\left(\neg \left(\left(\neg\left(\left(\neg K_{70} \oplus K_{11} \oplus K_{16}\right) \wedge \neg K_{28}\right) \oplus \neg K_{31} \oplus K_{69} \oplus K_{10} \oplus K_{15} \oplus K_{74}\right.\right.\right. \\
& \left.\left.\oplus K_{15} \oplus K_{20}\right) \wedge\left(\neg K_{44} \oplus K_{7} \oplus K_{10} \oplus K_{5} \oplus K_{11}\right)\right) \oplus\left(\neg \left(\left(\neg K_{61} \oplus K_{2} \oplus K_{7}\right)\right.\right. \\
& \left.\left.\wedge \neg K_{19}\right) \oplus \neg K_{22} \oplus K_{119} \oplus K_{60} \oplus K_{1} \oplus K_{65} \oplus K_{11}\right) \wedge\left(K_{76} \oplus K_{17} \oplus K_{22} \oplus K_{40}\right. \\
& \left.\oplus K_{3} \oplus K_{43} \oplus K_{9} \oplus K_{38} \oplus K_{1} \oplus K_{4} \oplus K_{5} \oplus K_{44} \oplus K_{7} \oplus K_{8} \oplus K_{5} \oplus K_{11}\right) \\
& \underbrace{\left.\oplus \neg K_{38} \oplus K_{1} \oplus K_{4} \oplus K_{5} \oplus K_{80} \oplus K_{21} \oplus K_{26} \oplus K_{43} \oplus K_{6} \oplus K_{46} \oplus K_{12}\right)}_{f_{177}^{\prime}}
\end{aligned}
$$

$\oplus / V_{0} \wedge \underbrace{\left(\neg K_{44} \oplus K_{7} \oplus K_{10} \oplus K_{5} \oplus K_{11}\right)}_{S_{160}^{177}}$

$\triangleright$ Attacking $y_{49}=f_{177} \oplus / V_{49}$ requires 2 hypotheses: $y_{49}=f_{177}^{\prime} \oplus / V_{0} \wedge S_{160}^{177} \oplus / V_{49}$

$\triangleright$ The more the state is updated, the more IV bits interfere in $f_{i}$

$\triangleright$ Still possible to isolate key-dependent variables but increases exponentially the attack complexity 


\section{Selection Functions $\varphi_{i}$}

$\triangleright$ Attacking $\varphi_{i}$ for $i \geq 82$ is sufficient to recover the entire key [AFM18]

\begin{tabular}{lc}
\hline \multicolumn{1}{c}{$\varphi_{i \in \mathcal{I}}$} \\
\hline$[0,48]$ & $f_{128+i} \oplus / V_{i}$ \\
{$[49,57]$} & $f_{128+i}^{\prime} \oplus\left(S_{160}^{128+i} \wedge / V_{i-49}\right) \oplus / V_{i}$ \\
{$[58,62]$} & $f_{128+i}^{\prime} \oplus\left(S_{160}^{128+i} \wedge / V_{i-49}\right) \oplus\left(S_{193}^{128+i} \wedge / V_{i-58}\right) \oplus / V_{i}$ \\
{$[63,96]$} & $f_{128+i}^{\prime} \oplus\left(S_{160}^{128+i} \wedge / V_{i-49}\right) \oplus\left(S_{193}^{128+i} \wedge / V_{i-58}\right) \oplus\left(S_{111}^{128+i} \wedge / V_{i-63}\right) \oplus / V_{i}$ \\
\hline
\end{tabular}




\section{Resulting Boolean System to Solve}

$$
\mathscr{F}_{[0,81]}= \begin{cases}\left(\neg K_{70} \oplus K_{11} \oplus K_{16}\right) \wedge \neg K_{28} \oplus K_{31} \\ \vdots \\ \left(\neg\left(K_{69} \oplus K_{10} \oplus K_{15}\right) \wedge \neg K_{27} \oplus K_{30} \oplus K_{127} \oplus K_{68} \oplus K_{9} \oplus K_{73} \oplus K_{19}\right) \wedge \cdots & =f_{128} \\ \left(f_{128} \oplus K_{69} \oplus K_{10} \oplus K_{74} \oplus K_{20}\right) \wedge S_{160}^{177} \oplus \neg\left(K_{61} \oplus K_{2} \oplus K_{7}\right) \wedge \neg K_{19} \oplus \cdots & =f_{176}^{\prime} \\ \vdots & =f_{177}^{\prime} \\ \left(f_{160} \oplus\left(\neg K_{44} \wedge \neg K_{2}\right) \oplus K_{5} \oplus K_{102} \oplus K_{43} \oplus K_{48}\right) \wedge\left(\neg K_{60} \oplus K_{1} \oplus K_{6} \oplus \cdots\right) \oplus \cdots & =f_{209}^{\prime} \\ \neg K_{44} \oplus K_{7} \oplus K_{10} \oplus K_{5} \oplus K_{11} & =S_{160}^{177} \\ \vdots & \vdots \\ K_{76} \oplus K_{17} \oplus K_{22} \oplus K_{39} \oplus K_{2} \oplus K_{42} \oplus K_{8} \oplus K_{37} \oplus K_{0} \oplus K_{3} \oplus K_{4} \oplus K_{43} \oplus \cdots & =S_{160}^{209} \\ \neg K_{86} \oplus K_{27} \oplus K_{32} \oplus K_{49} \oplus K_{12} \oplus K_{14} \oplus K_{52} \oplus K_{15} \oplus K_{18} & =S_{193}^{186} \\ \vdots & \vdots \\ \left(\neg K_{51} \wedge \neg K_{9}\right) \oplus K_{21} \oplus K_{109} \oplus K_{50} \oplus K_{55} \oplus K_{1} \oplus K_{72} \oplus K_{13} \oplus K_{18} \oplus \cdots & \\ \neg K_{9} & =S_{193}^{209} \\ \vdots & =S_{111}^{191} \\ \neg K_{27} & \vdots\end{cases}
$$




\section{From Theory to Practice - Experimental Setup}

$\triangleright$ 32-bit assembly implementation on an ARM Cortex-M3 (32 steps at once)

$\triangleright$ Fixed hard-coded 128-bit key $K=$ "Encryption Key K"

$\triangleright$ Electromagnetic emanations recorded using an industrial near-field probe

$\triangleright$ Correlation coefficient as statistical distinguisher and Hamming weight as leakage model

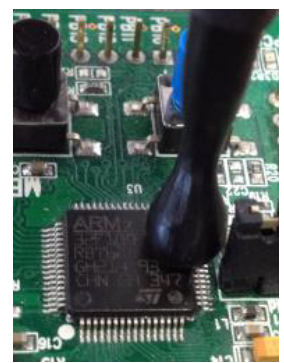

$\triangleright 5000$ encryptions using random IVs

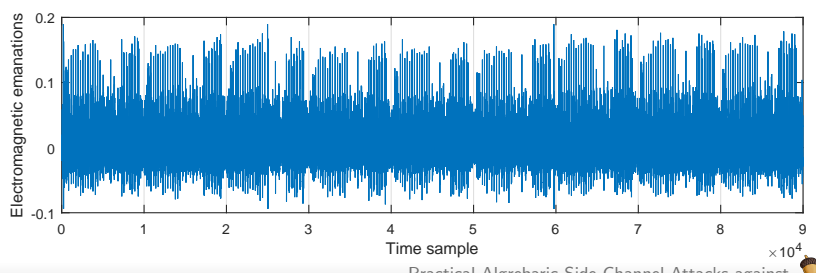




\section{From Theory to Practice - Successful Results}

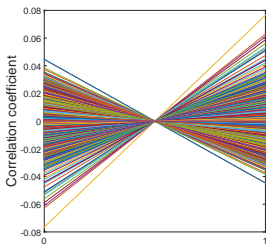

Key hypothesis

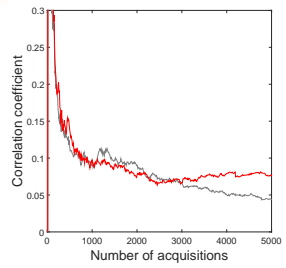

(a) $\varphi_{0}$ returns $f_{128}=1$
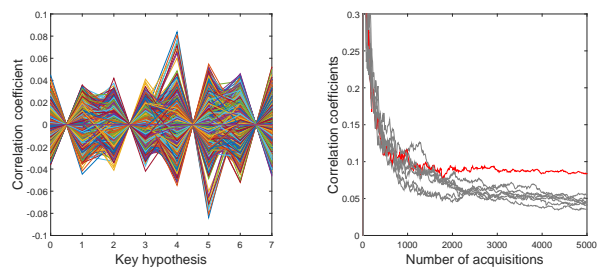

(c) $\varphi_{58}$ returns $f_{186}\left\|S_{160}^{186}\right\| S_{193}^{186}=(100)_{2}$ TRUSTED อBJECTS
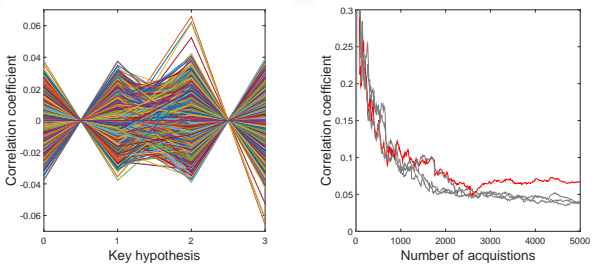

(b) $\varphi_{49}$ returns $f_{177} \| S_{160}^{177}=(10)_{2}$
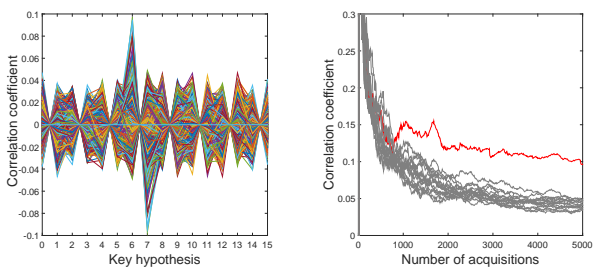

(d) $\varphi_{63}$ returns $f_{191}\left\|S_{160}^{191}\right\| S_{193}^{191} \| S_{111}^{191}=(0110)_{2}$ 


\section{Maximizing the Practical Efficiency (Opt. 1)}

$$
\varphi_{i \in[63,96]}(x, y, z, t)=x \oplus\left(y \wedge / V_{i-49}\right) \oplus\left(z \wedge / V_{i-58}\right) \oplus\left(t \wedge / V_{i-63}\right) \oplus / V_{i}
$$

$\triangleright$ Nonlinearity ensures a good distinguishability between correct and incorrect key guesses

$\triangleright$ Additional IV bits interact with hypotheses through bitwise AND

$\triangleright$ The more IV bits involved the more efficient the selection function is $\Rightarrow \varphi_{i \in[63,96]}$ requires less acquisitions for the correct hypothesis to stand out 


\section{Minimizing the Knowledge of Initial Vectors (Opt. 2)}

$$
\varphi_{i \in[0,48]}(x)=x \oplus / V_{i}
$$

$\triangleright$ Knowledge of full plaintexts/IVs is sometime unrealistic in practice $\Rightarrow$ interests in attack paths given the fewer bits to consider

$\triangleright$ ACORN defined by the concatenation of 6 LFSRs $\Rightarrow \varphi_{i \in[0,48]}$ can be used to recover up to 7 intermediate values $\left(f_{128+i}, f_{128+i}^{1}, \cdots, f_{128+i}^{6}\right)$

$\triangleright$ Knowledge of a single IV bit allow to recover up to 7 key bit combinations
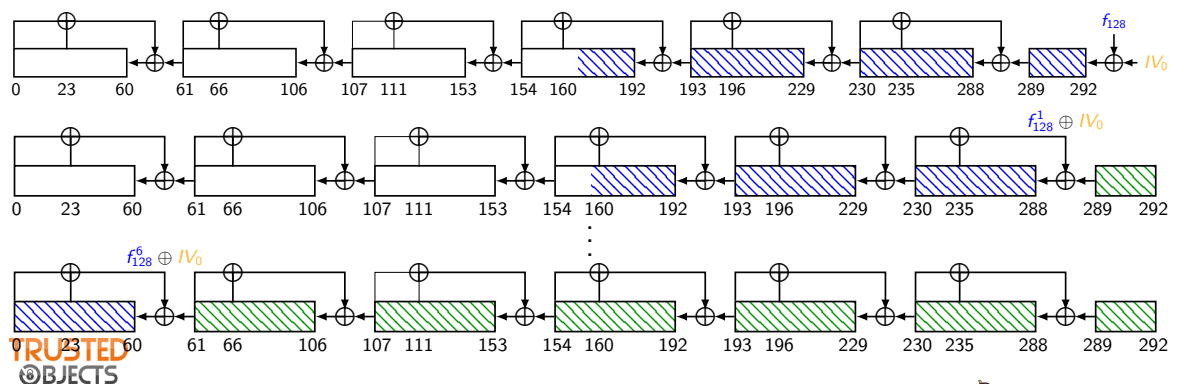


\section{Summary \& Comparison of our Practical Experiments}

$\triangleright$ Boolean systems were given as input to the SAT solver CryptoMiniSat 5.0 [SNC09]

\begin{tabular}{lccc}
\hline & Ref. & Opt. 1 & Opt. 2 \\
\hline IV bits to consider & $I V_{i \in[0,78]}$ & $I V_{i \in[0,95]}$ & $I V_{i \in[0,18]}$ \\
\# of required acquisitions & $\geq 4000$ & $\geq 2000$ & $\geq 4000$ \\
\# of attacked bits & 79 & 32 & $19 \times 6=114$ \\
\# of equations & 148 & 128 & 114 \\
\# of CNF clauses & 2165 & 1804 & 4251 \\
Solving time (i5-6200U CPU) & $0.05 \mathrm{sec}$ & $0.04 \mathrm{sec}$ & $87 \mathrm{~min} 17 \mathrm{sec}$ \\
\hline
\end{tabular}




\section{Conclusion \& Perspectives}

$\triangleright$ Practical full key recovery against ACORN using EM side-channels

$\triangleright$ Open the way for evaluations of its implementations on embedded devices

$\triangleright$ Introduced 2 optimizations regarding the reference attack

$\triangleright$ Some selection functions require to clearly identify point-of-interests (i.e. leakages in time) $\Rightarrow$ desynchronization countermeasures could be of great interest for this algorithm 


\section{References}

Alexandre Adomnicai, Jacques J.A. Fournier, and Laurent Masson.

Masking the Lightweight Authenticated Ciphers ACORN and Ascon in Software.

In Ferucio Laurentiu Tiplea and Bogdan Warinschi, editors, Cryptography and Information Security in the Balkans, Cham, 2018.

Springer International Publishing.

https://eprint,iacr.org/2018/708.

W. Diehl, A. Abdulgadir, F. Farahmand, J. P. Kaps, and K. Gaj.

Comparison of cost of protection against differential power analysis of selected authenticated ciphers.

In 2018 IEEE International Symposium on Hardware Oriented Security and Trust (HOST), pages 147-152, April 2018.

Mate Soos, Karsten Nohl, and Claude Castelluccia.

Extending SAT Solvers to Cryptographic Problems.

In Theory and Applications of Satisfiability Testing - SAT 2009, 12th International Conference, SAT 2009, Swansea, UK, June 30

- July 3, 2009. Proceedings, pages 244-257, 2009.

\section{Hongjun Wu.}

ACORN: A Lightweight Authenticated Cipher.

Submission to the CAESAR competition: https://competitions.cr.yp.to/round3/acornv3.pdf, 2014. 


\section{Thank you for your attention}

\section{경청해 주셨어 감사합니다}

